\title{
APPRAISING THE INFLUENCE OF STREET PATTERNS ON NON-MOTORIZED TRAVEL BEHAVIOUR IN AHMEDABAD CITY, INDIA
}

\author{
RONIKA POSTARIA \\ CEPT University, India
}

\begin{abstract}
Our cities have come a long way over time in terms of development. What is often ignored or taken for granted is the proportion of pedestrians and cyclists strolling around the city, who are always having to make compromises. It is important to know that there are variations in urban areas - the level of variety a street offers - each element defines the route options, which influences the travel pattern. It is therefore assumed that street patterns influence several aspects of non-motorized travel choices. This paper attempts to answer the question: "do the different assumptions regarding pedestrians and cyclists based on street pattern exhibit any modifications when tested on the ground (in Ahmedabad city)?" The research methodology consisted of various stages: in the initial stage, street patterns were differentiated based on the mathematical assimilation of their different characteristics and their respective influence on NMT behaviour was studied; in the later stage, surveys (a mixture of quantitative and qualitative questions) were conducted separately for pedestrians and cyclists. To reach the conclusions, the literature study and users' perception were assessed simultaneously. Various relations among different street patterns and non-motorized travel based on our understanding were formulated and tested in two ways, i.e. topologically and through on-ground users' preferences. Then, the test results were compared to reach the conclusion. The outcomes were different for both pedestrians and cyclists; there was no relation found between the street pattern and cyclists' travel choices. On the other hand, after looking at the results of both the tests, it was grasped that although pedestrians themselves were found to be unaware of the direct implications, their answers reflected how their choices were related to several aspects of the street pattern. The paper, therefore, concluded: 'street pattern influences several aspects of pedestrians' travel choices'.

Keywords: non-motorized travel choices, street pattern, pedestrians, cyclists, street topology, Ahmedabad city.
\end{abstract}

\section{INTRODUCTION}

Non-motorized transport includes several modes. This paper, however, focuses on walking and cycling as they are considered the most basic types. A number of policies emphasise the benefits and importance of Non-Motorized Travel, but most of the investments continue towards the advantage of cars.

What is often ignored or taken for granted is the proportion of pedestrians and cyclists strolling around the city. Our city plans do not look beyond the wide streets and required connections for cars. As a result, non-motorized travel users are always making compromises - whether it is space on road, the long distances, or the extreme weather conditions - no attention is paid to them. It is observed that people do not use Non-Motorized Travel (NMT) for different reasons and there are studies done to identify those issues. Nevertheless, no steps have been taken to address these issues in Indian cities, and work is done only on individual streets or small stretches. Most Indian cities have poor NMT infrastructure including poor lighting and narrow or fully absent footpaths. This paper, therefore, is focused entirely on non-motorized travel choices.

The case city selected was that of Ahmedabad, in the state of Gujarat. The city is no exception when discussing hospitability towards non-motorized transport users. Neither does 
the city have an attractive and clean environment for NMT users, nor does it have enough or even significant walking paths. Along with that, cyclists are expected to travel in the same lanes as motorised vehicles despite their slower speed and the limited space that they use up. The trends have remained static for years and no deliberate effort is put into solving these issues.

Moreover, this paper does not look at Non-Motorized Travel Choices in isolation. The study is an approach to understanding the underlying concepts of different street patterns, their significance, and their direct or indirect influence on people according to the on-ground conditions.

\section{HOW ARE STREET PATTERNS RELATED TO NON-MOTORIZED TRAVEL?}

The transport network is said to act as a base for the development of urban areas. While it affects our cities' growth in quite prominent ways, it also influences its users. The core purpose of road remains the same - movement. People make journeys to reach destinations, and the base network offers them options to reach those places. Moreover, the options provided may vary based on the mode of transport (private car, public transport, or nonmotorized transport). Since walking is considered an integral part of a journey, it often exhibits diversity with respect to the purpose of the journey as well as the user. The diversity is observed in terms of the choices (often varying according to different groups of users). Similarly, cycling has its own prerequisites for its users, and affects people's choice of mode. The variations in the urban context such as network, environment, connectivity, and the indirect relation to other people play an important role in the decision making process in the choosing of different modes of transport. It was thus assumed that street patterns influence several aspects of non-motorized travel choices.

Lewis Mumford once said, "no city can solve its transportation problem if it neglects the greatest, self-propelled vehicle of all: The Pedestrian" [1]. This statement can be related to our cities' present condition. While we all know, non-motorized travel is one of the most used, it is often not seen to be as important as it actually is. If we look at it the other way, it can be considered that the level of variety a street offers attracts different people, which then creates the mingling complex. Starting from the number of blocks, intersections, and access points until the very end of loops or cul-de-sacs, each element defines the route options differently, thus influencing the travel pattern. Therefore, this paper attempts to answer the question, "do the different assumptions regarding pedestrians and cyclists, made based on street pattern, exhibit any modifications when tested on-ground?"

\section{UNDERSTANDING STREET PATTERNS}

Settlements are made of people. Humans require many things to survive in the world, such as water, energy, information, etc., which flow back and forth between people through mutual networks. However, movement of people in itself is also very important. This particular movement often occurs through the transportation network - the most visible of all networks. Being evident on plans, the road networks tend to create a picture of our cities. Beginning with a number of roads and their total length, followed by their arrangement and spatial expansion from the city core, and at last the structures they form, all features give us a reflection of the transport network in the city, which, when schematically studied, can be distinguished in terms of the patterns formed.

A pattern, in simple terms, can be defined as a property of objects wherein different features such as shape, orientation, distribution, etc., are repeated, while maintaining regularity. The pattern of networks in any city develop in response to the change in size, 
shape, or physical constraints. Cities act as a circulation system for the larger commercial trade around the world. In that larger picture, streets become the smallest element. They make up for more than $50 \%$ of all public space in cities, however, the movement function - from the micro scale of circulation within buildings to the macro scale of whole cities - remains central in the context of spatial organisation. Street classifications are usually based on traffic- or urban-function (activities on the street, for example). As a matter of fact, on one particular street, a variety of characteristics are present, thus giving the street 'multiple personalities' [2]. Due to such complexities, attempts to isolate and codify the variations in patterns are varied [3], yet not clearly classified. The recognition and representation of the several street patterns have a certain tenacity, and it often changes in accordance with the several typological sets. In the words of Stephen Marshall, 'it depends on the eye of the beholder' [2].

\subsection{Understanding patterns' formation}

The original unplanned street networks, have evolved and shall keep on evolving over time. The street network, quite simply, can be described as interconnecting lines and points, which, in urban planning terms, are referred to as "links and nodes". The overall structure formed represents the street layout which acts like the skeleton of the city plan. Every city, by default, has a different street layout; some influenced by physical barriers, some by topography and some others by the mere expansion of the city. However, many of them may have followed a similar process of transformation from ancient times to the modern era. When analysed, it may be observed that most street networks do not have a unique growth trend. Indeed, a small part of the layout structure is repeated several times, in different manners to form a larger network. That particular aspect, which appears on several different networks, is defined as the street pattern.

The problem that urban planners face today is the choice of pattern for planning the transportation network. There is no good or bad pattern described because street patterns do not exist in isolation. Various other aspects, including geographical features of the city, land use distribution around the city, and the typical modal share - all of which vary according to place and time - influence the street patterns. However, the usual methods of planning follow a system of imposing a few good examples repeatedly. But the problem with such an approach is that what works in one place might prove to be a disaster in another location. It, therefore, becomes important to understand the process that led to the formation of a particular pattern and the resulting characteristics.

Stephen Marshall, in his book "Streets and Patterns", tried defining three analytical levels following the 'part-to-whole' approach. It is commonly referred to as 'The Three Cs' Composition, Configuration, and Constitution. While composition deals with basic geometric aspects of roads, such as length, width, the angle of orientation, etc., Configuration looks into the conceptual aspects of links and nodes, their placement and ratio, etc. Moreover, Constitution deals with elements of the larger structure. It considers the hierarchy and relation of the structural elements.

Nevertheless, all of them have one common element - "the street".

\subsection{Streets to patterns}

Christopher Alexander, in The Pattern Language, stated, "Each pattern is a three-part rule, which expresses a relation between a certain context, a problem, and a solution" [4]. 
When described in relation to street patterns, the statement reflects the three central aspects of transportation planning. The context is the building frontage which defines the character of the street; the problem reflects the issues of connectivity and accessibility which is the actual purpose of the street network; and the solution lies in understanding the relation of such street features (as connectivity, accessibility, etc.) to the street pattern itself.

The mentioned relation can only be understood if each characteristic is properly known. However, the understanding of street pattern itself is very difficult. This is because 'patterns' cannot be defined in any standardised manner. The descriptions are often too vague or too specific as the variety of patterns is abundant while the description varies from person to person.

In "Streets and Patterns", Stephen Marshall suggested a few structural properties of the different aspects of the hierarchical layout. The properties are: differentiation of components; ordered ranking of elements; necessary and allowable connections between different types of elements; frequency distribution; and structural configuration of the elements. The references are made such that the structure of the road network is compared to that of a tree (referred to as the 'tree analogy'). Marshall also suggested a street-based Constitution wherein, the transport pattern can be linked to the land use pattern.

Modes, Arteriality, Access Constraint and Connection Type together create a system for Transport network management while Frontage use helps in creating a street type which together with above four aspects creates a system for generating a street pattern [2].

\section{ANALYSIS AND FINDINGS}

To reach conclusions, it was important to assess the literature study and users' perception simultaneously. Since the literature study (and case studies mentioned) dealt with pure street pattern types, they could not be applied entirely for a hybrid (a mixture of several types) street pattern like that of Ahmedabad. It could be rationalised only after considering the perception of users, and how they relate to the street pattern.

\subsection{Ahmedabad city's street network and transportation}

The street network in the old city is amorphous, proving the efficiency of ancient street patterns. As the city grew slowly spreading vastly in all directions, the city planners tried implementing ring-radial road network. The present city has about 20 well-defined radials, and several rings [5].

As a matter of selection, the initial idea was to work on separate neighbourhoods across the city. However, a neighbourhood scale is often open to interpretations. In order to keep the scale local as possible and yet large enough to reflect a distinct street pattern, the scale 'ward' was chosen. This not only simplified the work but also gave us the liberty of studying the interrelations of the various possible choices of non-motorized travel being influenced by different, yet similar, factors. Thus, five different wards (of varying scales) were selected, whilst two major criteria were considered. First, the street pattern should relate to one of the "pure-types" (linear, radial, tree, grid), and second, the surrounding land use should be understood to know about the trip attractors and trip-generators.

\subsection{Understanding cyclists' travel choices in Ahmedabad}

Many researchers claim that non-motorized travel (NMT) in developing countries is often much more than a choice. As we go across the income strata in India, it becomes clear that cycling is often a preference due to lack of any other affordable alternatives. There are also 
people who use it for recreation or fitness purpose, though the comparison would be in fractions. For this reason, the survey samples remained the same across all the wards.

When categorised as per age group across all the five wards, it was observed that both male and female, though in different age-groups, are daily users of bicycles. The only exception were females in the age group $>60$ years, in which cycling was found to be totally absent.

Further, when asked 'How often do you cycle and why?', the answers were as shown in Fig. 1. only children used bicycles for recreational purposes. Most people who cycled daily had 'no other choice' resulting into $58 \%$ of the reasons for cycling. This category mostly consisted of people belonging to lower-income groups, where males commuted across the wards throughout the city for various different types of work. Females, on the other hand, either worked as house cleaners or used cycles to pick up/drop off their kids or siblings. Moreover, a small proportion, $22 \%$, used cycles because they 'prefer it over vehicles'. These were among the age group $<13$ years and majorly youngsters from the age group 13-35 years.

While the questionnaire was conducted, there were several questions asked to the cyclists. One of those was "What influences you while cycling?" This question was an important one as it helped us in identifying if cyclists in Ahmedabad are influenced by street patterns in any way. The recorded answers are shown in Fig. 2. After looking at the answers, we were unable to find the relation to street pattern. As an alternate option, we also looked at answers to questions like "Do you face any issues while cycling?", "What can be better?", and "When would you cycle more?" However, all of the answers (not the majority - all) were highlighting the fact that cyclists are not given any importance in Ahmedabad city. They complained about their experiences, suggested some things government can do and talked about how and why certain things are important for them, but nobody mentioned anything relating to Street Pattern. They were all mostly concerned with their experiences and the attention required to meet their needs, that the aspects of preference and choice were almost missing.

We came across some important concerns of cyclists in Ahmedabad out of which most remain unnoticed by the planning committees or governing bodies. However, the hypothesis of the research, "Street Pattern influence several aspects of Non-Motorized Travel Choices" was stated falsely in the case of cyclists, leaving no room to find answers to the research question.

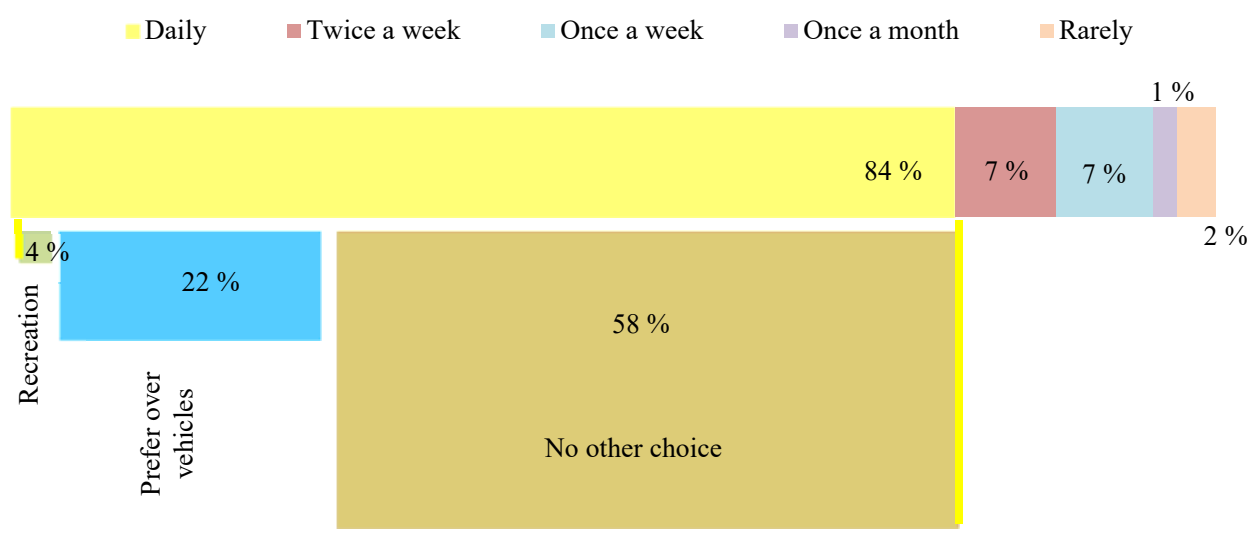

Figure 1: Cyclists' frequency and reasons. 


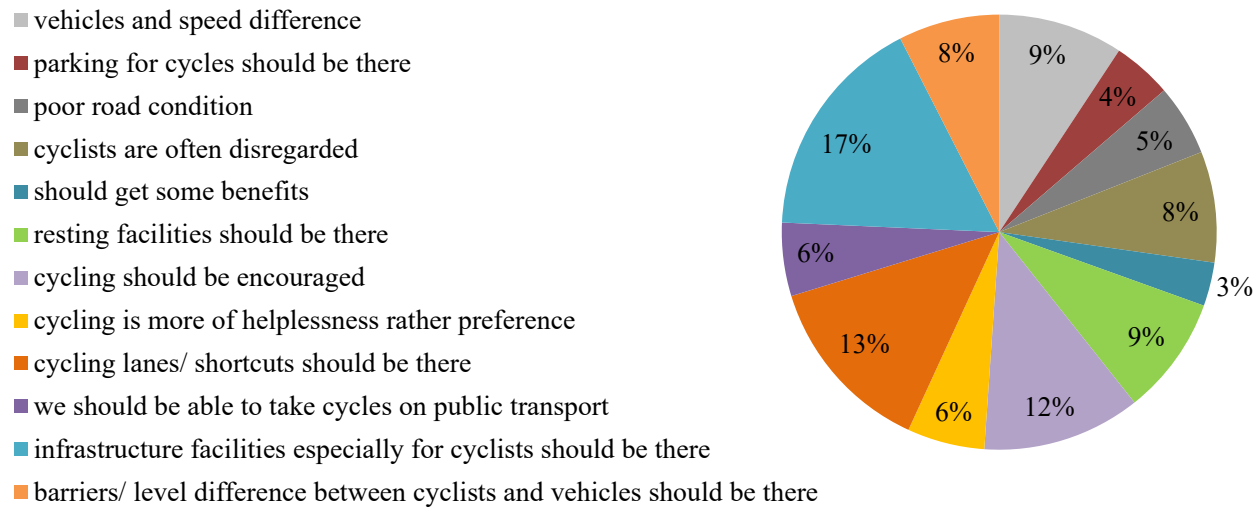

Figure 2: Recorded answers for "What influences you while cycling?".

\subsection{Understanding pedestrians' travel choices in Ahmedabad}

Pedestrians in Ahmedabad do not have the comforts of quality infrastructure and an encouraging environment. Before beginning the survey, a small observatory study was done to identify the major pedestrian routes and time slots. In order to collect a variety of opinions from people with different purposes and preferences, the survey was done in each ward in two-time slots, i.e. morning and evening.

Although the data collected has detailed opinions of people regarding their choices, convenience, and expectations. The detailed analysis demanded inter-linking the factors in order to observe the influence of street pattern. The first step was to understand the nature of the surveyed target group, identifying the similarities and differences to answer the first basic question, "Do street pattern influence their choices?"

While we tried to bifurcate among the age groups and genders, it was observed that the number of youngsters was higher in all the wards while the number of children was lowest. Further, the segregation among males and females remained equal throughout.

We then moved on to identifying their walking frequency and the reasons behind those choices, i.e. how often do they walk, and why do they walk? People do walk every day for one reason or another. The mode share of walking as daily commuting mode was quite balanced. While some wards presented us with equal proportions of answers for 'prefer over vehicles' and 'no other choice', others suggested equal proportions for 'recreation' and 'no other choice'. Overall, the proportion of walking for recreational purposes was the choice of only one-fifth of the pedestrians.

The next task was to know if the street pattern plays any role in pedestrians' choices or not. Considering the potential and limitations of open-ended questions, there was a range of answers recorded. They were first segregated for individual wards as they were in raw data, and then the average was observed across the wards. A problem faced was that the influence of street patterns might not always be dominant for the pedestrians. Being a planner or designer, we perceive things quite differently than the users. So, the question of whether street patterns influence them or not, was not asked directly; rather we answered it from interpreting their answers to several different questions such as "Do you face any issues while walking?", "When would you walk more?", and "What can be better?" The answers then were categorised based on their relation to street topology and pattern. 
It was observed that almost half of the answers related to street patterns in wards like Bodakdev, Naranpura, and Navrangpura. Answers recorded in Paldi and Jamalpur, on the other hand, were around 35\%. However, what influences the pedestrians and how is it connected to street patterns was very clear.

\subsection{Examining the assumptions}

Through objective one 'Understanding Street Patterns', the different characteristics' underlying concepts provided several attributes that could be detailed and used for the research. Moreover, the basis of differentiation of pattern formations and their corresponding types helped in creating a strong base for the research study. Progressing towards the findings of objective two 'Understanding user perception in accordance with on-ground conditions', it was understood that different patterns influenced cyclists and pedestrians differently. Further, it was found that although pedestrians are influenced by street pattern, the cyclists in Ahmedabad are not.

Objective three 'Identifying similarities and differences with regard to literature, and reviewing the reasons for the same', was attained through reviewing several case studies having a similar or relatively similar aim to us, and various relations among different street patterns and non-motorized travel based on our understanding were formulated (these assumptions were formed under objective 1). Those assumptions were then tested (in objective 3) in two ways to attain the final answer to the research question. Firstly, they were tested topologically, i.e. through looking at various aspects of street pattern formation, it was to be determined whether the assumptions were true or false. In absence of direct relations, we went back and forth to understand how certain things do not happen in absence of some important aspects of street pattern. The second method returned to our on-ground users and their answers recorded during the surveys. Then, the test results through both ways were compared to reach the conclusion.

The purpose was not to prove that they contrast with each other but to understand why they are not same.

\subsubsection{Assumption 1: Grid pattern is most walkable as it gives route options}

There were two key words, i.e. 'Walkable', and 'Route-options'. Any area is considered to be walkable when people have various options to reach their destinations but the distance from the origin to destinations remains the same or similar.

Topologically, we could measure this in terms of 'the ratio of shortest and longest route distance' as shown in Fig. 3. To understand people's perception they were asked, “Are places accessible by walking?" as shown in Fig. 4.
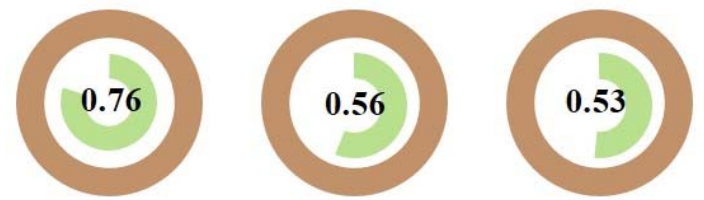

Shortest distance

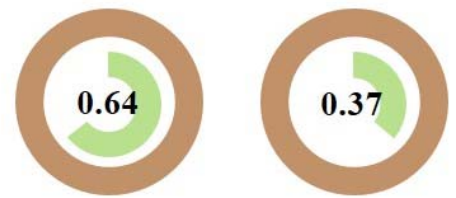

Longest distance

Figure 3: Shortest to longest distance ratio for Bodakdev, Naranpura, Navrangpura, Paldi, and Jamalpur respectively (from left to right). 

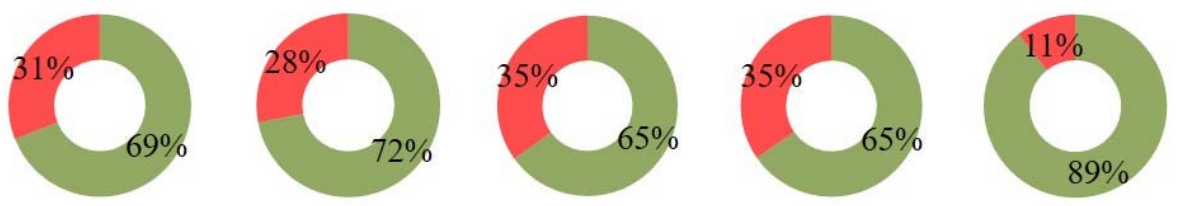

No

Yes

Figure 4: People's perception of "Are places accessible by walking?" in Bodakdev, Naranpura, Navrangpura, Paldi, and Jamalpur respectively (from left to right).

According to topological test, the walkability was found out to be highest in Bodakdev (grid), followed by Paldi (tree-like), Naranpura (grid), Navrangpura (tree-like), and least in Jamalpur (organic). The results suggest that the assumption was true for all other wards but Paldi. However, pedestrians' perception stated a different result where the assumption was proved true for all wards but Jamalpur (possible explanation could be the lack of route options).

Moreover, considering the ratio of 1:4, we stated that the assumption could be taken to be correct in most cases, particularly in cities where a hybrid pattern is present.

\subsubsection{Assumption 2: Tree-pattern is unsafe due to higher vehicular speeds}

The key words here were 'Unsafe', and 'Vehicle-speed'. Vehicles speed up when they have wide roads or empty roads for long. If we consider their collision with other vehicles, they are bound to slow down.

Topologically, as shown in Fig. 5, we could measure this in terms of 'number of junctions'. To understand people's perception they were asked, "Do you feel safe from vehicles while walking?" whose answers are shown in Fig. 6.

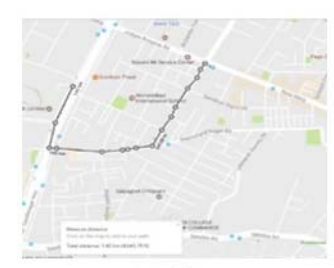

$1.3 \mathrm{~km}$ 16 junctions

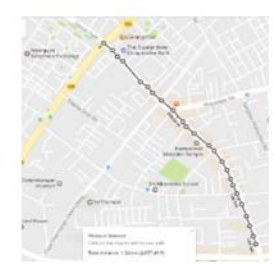

$1.3 \mathrm{~km}$ 25 junctions

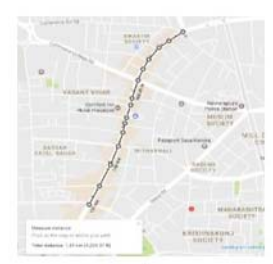

$1.3 \mathrm{~km}$

15 junctions

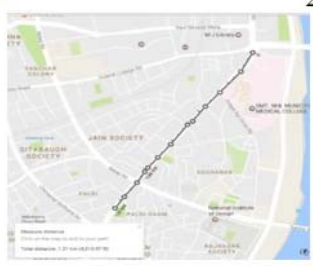

$1.3 \mathrm{~km}$

13 junctions

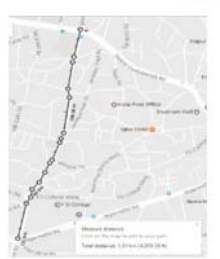

$1.3 \mathrm{~km}$ 16 junctions

Figure 5: Number of junctions on major roads in Bodakdev, Naranpura, Navrangpura, Paldi, and Jamalpur respectively (from left to right). 

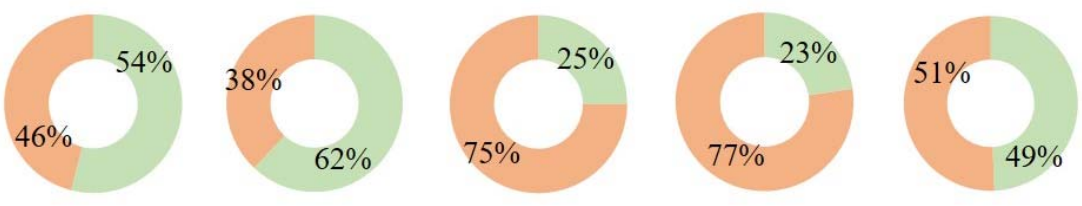

No

Yes

Figure 6: People's perception of "Do you feel safe from vehicles while walking?" in Bodakdev, Naranpura, Navrangpura, Paldi, and Jamalpur respectively (from left to right).

Topologically, Paldi (tree-like) was found to the most unsafe, followed by Navrangpura (tree-like), Jamalpur (organic), Bodakdev (grid), and Naranpura (grid) as most safe. The assumption thus proved true for all the wards other than Bodakdev, which has a grid pattern but has a number of junctions equivalent to Jamalpur and Navrangpura. Further, looking at the recorded answers of the pedestrians, it was observed that Paldi and Navrangpura were considered to be unsafe while Bodakdev, Naranpura, and Jamalpur were considered to be safe. The assumption was thus proved true for all the wards despite the topological results.

\subsubsection{Assumption 3: Vehicle-pedestrian collisions are less when there are more of T-} junctions and less of $\mathrm{X}$-junctions

There were two key words, i.e. 'Vehicle-pedestrian collisions', and 'X- and T-junctions'. There are less of conflict points when there are less of roads.

Topologically, X-and T-junctions together with Cul-ratio and Cell-ratio were calculated for patterns and tested in a 2-D graph as shown in Fig. 7. To understand people's perceptions, they were asked a question such as "Why not?" when they answered 'No' in terms of safety from vehicles and the corresponding answers were then sorted in terms of those relating to 'vehicles at junctions' as listed in Fig. 8.

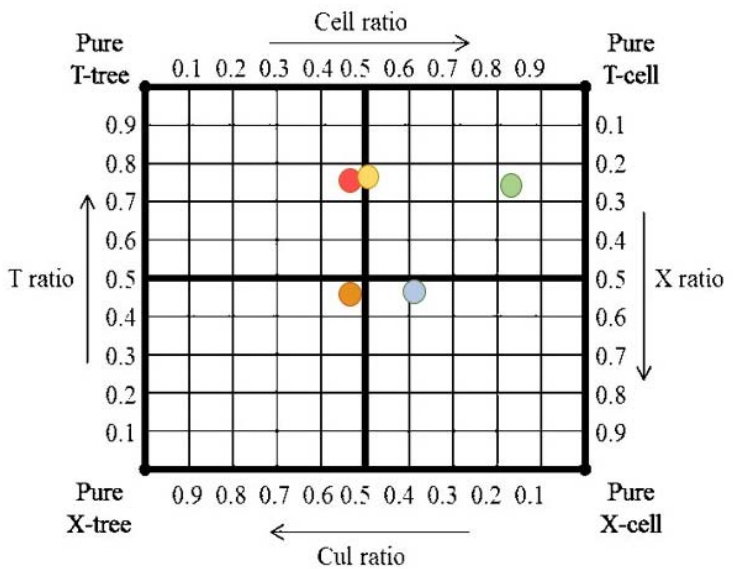

Bodakdev

No. of X-junctions: 274

No. of T-junctions: 246

Naranpura

No. of X-junctions: 121

No. of T- junctions: 119

Navrangpura

No. of X-junctions: 79

No. of T- junctions: 270

Paldi

No. of X-junctions: 103

No. of T- junctions: 333

Jamalpur

No. of X-junctions: 20

No. of T-junctions: 76

Figure 7: Number of junctions and the ward's pure pattern. 

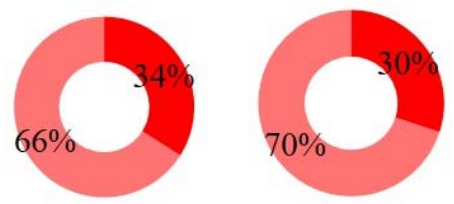

Junctions are unsafe
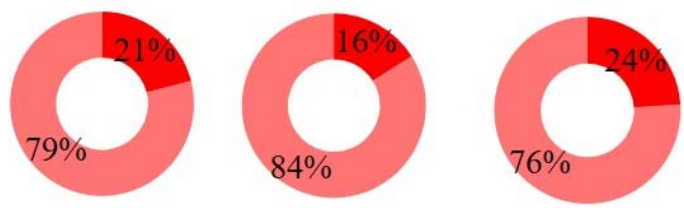

No emphasis on junctions

Figure 8: People's perception of 'How many people think safety from vehicles is not there at junctions' in Bodakdev, Naranpura, Navrangpura, Paldi, and Jamalpur respectively (from left to right).

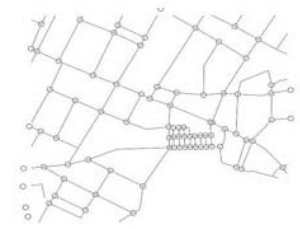

Intersections: 74 | Nodes: 83 Connected-nodes ratio: 0.89

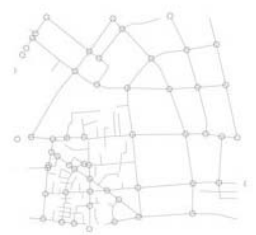

Intersections: 52 | Nodes: 69 Connected-nodes ratio: 0.75

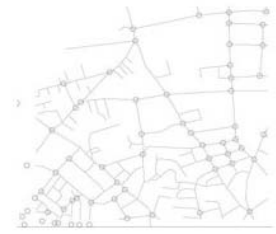

Intersections: 59 | Nodes: 119

Connected-nodes ratio: 0.49
Intersections: 74 | Nodes: 112

Connected-nodes ratio: 0.66
Intersections: 48 | Nodes: 56

Connected-nodes ratio: 0.85

Figure 9: Connected-Node ratio (per unit area) $(1.5 \times 1.5 \mathrm{~km})$ for Bodakdev, Naranpura, Navrangpura, Paldi, and Jamalpur respectively (from left to right).

Here, the assumption was proved true for all the wards. People felt that junctions were unsafe due to vehicles mostly in Bodakdev, followed by Naranpura, Paldi, Navrangpura, and Jamalpur. The comparative ratio of those feeling unsafe in a ward with a grid pattern and those in wards with tree pattern was clearly visible to be high.

\subsubsection{Assumption 4: Grid pattern allows vehicles to spread across all streets irrespective of hierarchy}

The key words here were 'Vehicles', and 'Hierarchy'. If vehicle users have alternate options, where the traffic would be less or if the street acts as a shortcut, most of them tend to take those routes even if they are narrow or used for some other purpose. 

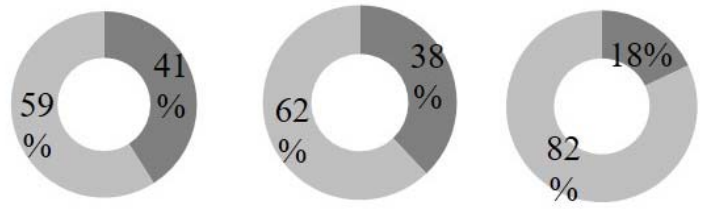

Threat from vehicles
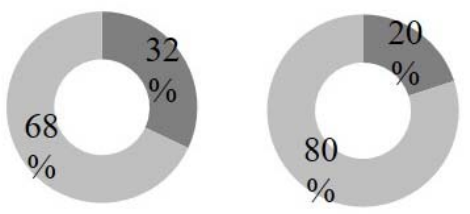

Does not influence

Figure 10: People's perception of "Do vehicles influence you while walking?" in Bodakdev, Naranpura, Navrangpura, Paldi, and Jamalpur respectively (from left to right).

Topologically, we could measure this in terms of 'connected-node ratio' as shown in Fig. 9. To understand people's perception 'issues faced by pedestrians' were studied, and those relating to vehicular traffic or speed were segregated as listed in Fig. 10.

The results of the topological test were such that Bodakdev was found to be most connected followed by Jamalpur, Naranpura, Paldi and Navrangpura; and therefore, so was the order of options vehicles have to move across the ward. However, when we looked at the people's perception, we found that "threat from vehicles" was also highest in Bodakdev, followed by Naranpura, Paldi, Jamalpur, and Navrangpura. The assumption thus was true in both the cases.

\section{CONCLUSION}

The outcome of the research had been quite promising. Through in-depth study of the topic, understanding it through the use of literature and also considering the on-ground activities, we achieved the stated aim of the research. Since we treated 'walking', and 'cycling' as entirely different modes, the conclusions were also drawn differently.

It was observed that cyclists in Ahmedabad, at this point in time, have more concerns regarding infrastructure facilities and comfort rather than convenience and preference (which were expected to relate to street patterns). Where there is no great importance given to the cyclists in terms of either space or infrastructure, they are more concerned about being recognised and addressed through visible attempts at easing their travel. The research question, therefore, was no longer applicable. Therefore, the topological testing was not done. This paper highlights the important aspects that influence the cyclists other than Street Pattern, however, there was no relation found between the Street Pattern and their travel choices.

On the other hand, after looking at the results of the tests, topological perception, as well as users' perception, it was found out that Street Pattern does influence the Pedestrians. Although several expectations about who walks and why they do so were contrasting for the on-ground users and their choices; there was still little connection to why is it important to consider them along with other modes of transport. In addition, pedestrians themselves were found to be unaware of the direct implications, but their answers reflected how their choices were related to several aspects of Street Pattern. Hence as the paper assumed 'Street Patterns' influence several aspects of "Non-Motorized Travel Choices" - the hypothesis was proved correct. Further, it was observed, that the assumed relations among street pattern and NMT users when tested topologically proved to be majorly true with few exceptions. However, when they were analysed from users' perspective, the results were 50-50. There were some 
assumptions entirely true in the case of pedestrians' preferences while it was false topologically for some, and there were also results where topologically results were promising but pedestrians' concerns stated otherwise.

The research, therefore, concluded, "Street Pattern influences several aspects of Pedestrians' Travel Choices". In addition, the Street pattern is only one aspect, and unlike vehicles, pedestrians consider several other aspects too. Thus, although the assumed influence exists, the pre-assumptions regarding their relation or the reasons for those influences are not always true.

Since the road network is the foremost dominating aspect of our city plans, it is important for planners to consider all the users rather than only motor vehicles. Our road networks are bound to be planned so that more and more cars are accommodated; vehicle users have multiple options to reach their destinations, and they have access to wherever they need to go. However, never is it thought that street patterns, or most commonly referred to as Street Layouts (which are actually different considering the scale), may also influence the lowest strata of transportation, i.e. walking and cycling.

Now that the results are quite promising, at least in the case of pedestrians, it can be of real help if considered at the right time. While the Ahmedabad city government fails to respond to the required infrastructure facilities, even footpaths at the lowest level, they can at least try to encourage walking in any way possible. There is also a need to identify cycling as an important mode of transport and consider the users' requirements. However, the scope of this research is limited up to understanding and analysing, and no recommendations were given as a result. The sole purpose was to explore the underlying relation between Street patterns and Non-Motorized Travel, which is not visible physically but exists somewhere behind the veil of the beautified cityscape.

\section{ACKNOWLEDGEMENTS}

I would like to express the deepest appreciation to my mentor Abhijit Lokre, who has the attitude and the substance of a genius. He continually and convincingly conveyed a spirit of excitement regarding exploration of the topic.

In addition, a thank you to Professor Shivanand Swami, who reviewed my work consistently and suggested the possible ways of attaining the aim of the research.

I would also like to thank the CEPT University for allowing me to work on this dissertation, use the multiple resources in the library, and explore my knowledge.

\section{REFERENCES}

[1] Mumford, L., The Highway and the City, Greenwood Press, 1981.

[2] Marshall, S., Streets and Patterns, Routledge, 1996.

[3] Eisner, S., SEAG, The Urban Pattern, John Wiley and Sons, 1993.

[4] Alexander C., MSSI, A Pattern Language: Towns, Buildings, Construction, Oxford University Press, 1977.

[5] CEPT University, Bus Rapid Transit System Plan, Ahmedabad: AMC (Ahmedabad Municipal Corporation), (COE) Centre for Excellence in Urban Transport, 2008. 